\title{
Tumour necrosis factor inhibitors and infection: What is there to know for infectious diseases physicians?
}

\author{
BL Johnston $M D^{1}$, JM Conly $M D^{2}$
}

$\mathrm{A}^{\mathrm{t}}$ this year's meeting of Digestive Disease Week in Los Angeles, California, it was noted that antitumour necrosis factor (anti-TNF) inhibitors showed further evidence of efficacy in Crohn's disease (1). TNF antagonists have been used increasingly in the treatment of a number of other inflammatory diseases, especially rheumatoid arthritis (RA), where they have revolutionized treatment (2). At the same time, their use has been associated with an increased risk of serious infections (3). As more patients are treated with these agents, it is inevitable that infectious diseases physicians will see a variety of infections, both common and uncommon, requiring familiarity with this new class of drugs and their infectious complications.

Cytokines, such as TNF- $\alpha$, are produced by activated monocytes, macrophages and $\mathrm{T}$ lymphocytes, and are a key component of the inflammatory response that leads to tissue damage (2). It is therefore intuitive that researchers would explore the ability of cytokine antagonists to have a therapeutic benefit in inflammatory disorders. Initial animal studies have pointed to a role for TNF- $\alpha$ in the pathogenesis of RA and the beneficial effect of anti-TNF antibodies (2). It has now been more than 10 years since evidence of the improvement seen in RA patients given a TNF-inhibitor was first reported (4). Since that time, several TNF- $\alpha$ antagonists have been developed and licensed for a number of indications.

The TNF- $\alpha$ antagonists currently licensed for use in Canada are infliximab (Remicade, Schering-Plough, USA), etanercept (Enbrel, Amgen, USA) and adalimumab (Humira, Abbott Laboratories, USA). Infliximab and adalimumab are monoclonal antibodies. Infliximab has a murine variable (antigen-binding) region and a human constant region, making it a chimeric monoclonal antibody (5). Adalimumab is a recombinant human monoclonal antibody (2). Neither of these agents are known to bind to any antigen other than TNF (3). Etanercept is a dimeric fusion protein with a human TNF receptor connected to the $F_{c}$ portion of human immunoglobulin G1 (6). In addition to TNF, etanercept also binds lymphotoxin-alpha (6). Both etanercept and adalimumab are administered subcutaneously, while infliximab requires intravenous infusion (2). All three drugs have fairly long halflives: four days for etanercept, nine days for infliximab, and two weeks for adalimumab (2). Their molecular differences may have clinical implications in terms of efficacy and safety (4).

The established indications for TNF- $\alpha$ antagonists have been for the treatment of certain patients with RA and Crohn's disease (7). They have subsequently been used in a variety of other conditions, both on- and off-label: ankylosing spondylitis, juvenile RA, psoriatic arthritis, acute alcoholism-associated hepatitis, Behçet's disease, psoriasis, uveitis, pyoderma gangrenosum, Still's disease, sarcoidosis, Sjögren's syndrome and post-stem cell transplant graft versus host disease $(7-10)$.

Randomized, controlled trials comparing the safety and efficacy of the TNF- $\alpha$ antagonists with that of standard therapy and/or placebo have demonstrated a similar frequency of infection $(2,5,10)$. This tends to be the case for adverse events that are rare and unlikely to be identified in these relatively small trials. On the other hand, the literature is rife with case reports and small case series describing the latest unusual infections in patients being treated with TNF- $\alpha$ antagonists. While these reports are interesting, they cannot answer the question of whether these infections are a direct result of the TNF- $\alpha$ antagonists or are secondary to immunosuppression from the underlying disease or other therapies (2). The answer to this question will rely on larger databases, including meta-analyses and postmarketing studies.

There is a strong theoretical basis to support an increased risk for infection with the use of TNF- $\alpha$ antagonists (11-13). The proinflammatory role of TNF, while a negative factor in disease pathogenesis, is important in the host immune response to a number of microbial pathogens. Several authors $(12,13)$ have reviewed the role of TNF in Mycobacterium tuberculosis infection, summarizing its activity in the immune response. TNF is produced by activated monocytes, macrophages and lymphocytes in response to a number of different pathogens, including viruses, bacteria and parasites, with biological effects including antitumour and antiviral activity, and mediation of shock and cachexia (12). These effects occur through apoptosis, cell activation, induction of other inflammatory cytokines and recruitment of inflammatory cells to sites of infection (7). TNF acts with other cytokines, especially interferon-delta, to generate a competent cellmediated immune response to intracellular pathogens such as M tuberculosis, Listeria species and Histoplasma species (7). This response leads to granuloma formation, which is the hallmark of mycobacterial infections and key to the successful control of infection (13). The importance of TNF- $\alpha$ in the host response against tuberculosis has been well demonstrated in animal models (12). Its protective role in the human response to mycobacteria is less clear but is implied given the increased numbers of tuberculosis (TB) cases seen with the use of the TNF- $\alpha$ antagonist infliximab (14).

${ }^{1}$ Queen Elizabeth II Health Sciences Centre and Dalhousie University, Halifax, Nova Scotia; ${ }^{2}$ Departments of Pathology and Laboratory

Medicine, Medicine, and Microbiology and Infectious Diseases, Centre for Antimicrobial Resistance, University of Calgary, Calgary, Alberta

Correspondence and reprints: Dr BL Johnston, Queen Elizabeth II Health Sciences Centre, 5014ACC - 1278 Tower Road, Halifax, Nova Scotia

B3H 2Y9. Telephone 902-473-5553, fax 902-473-7394, e-mail ljohnsto@dal.ca

Received and accepted for publication July 24, 2006 
TB has been reported in association with all of the TNF- $\alpha$ antagonists and has been the most common serious infection seen with their use $(2,3,7)$. The first case of active TB during treatment with infliximab was published in 1999 (14). Keane et al (15) published a review of data from the Food and Drug Administration's Adverse Event Reporting System (AERS) in 2001. Based on manufacturers' data, the investigators found that 147,000 patients received infliximab and 102,131 patients received etanercept during the study time frame. They also found that 70 patients (24.4 per 100,000 treated) developed tuberculosis during or after infliximab administration at a median of 12 weeks from the start of treatment. The background rate of TB in United States (US) patients with RA was calculated at 6.2 per 100,000 RA patients (95\% CI 0.6 to $34.0)$. In this cohort, $56 \%$ of patients had extrapulmonary disease and 24\% had disseminated disease. There were only nine reported TB cases among patients on etanercept, with no details reported on the patients. The authors concluded that, while not proven, the association between the use of infliximab and reactivated TB was strong. Postmarketing surveillance of etanercept has estimated a TB rate of approximately 17 per 100,000 treated patients worldwide and approximately 13 per 100,000 treated US patients $(16,17)$. Based on similarly acquired denominator data, the approximate TB rates for infliximab- and adalimumab-treated US patients were 50 per 100,000 and 20 per 100,000, respectively (17). Comparison of these rates must be done with caution. The cases are reported by passive surveillance, thus creating a reporting bias. Patterns of specific TNF use, as well as endemic TB rates, vary from country to country, making the rates not directly comparable. Despite these caveats, it appears that the risk of TB is indeed lower in patients receiving etanercept (12). This impression was supported by a later study using the AERS and looking only at patients treated in the US (18). In this study, TB was reported in approximately 144 per 100,000 infliximab-treated patients and approximately 35 per 100,000 etanercept-treated patients $(p<0.001)$. There were insufficient numbers of patients on adalimumab to analyze its infection rate. The median time to the onset of adverse events was 40 days for infliximab and 236 days for etanercept $(\mathrm{p}<0.001)$.

It is thought that this difference in infection rates is related to the differing molecular structures and activities of the two drugs. Infliximab, but not etanercept, has been shown to cause apoptosis, which may lead to the elimination of memory cells that lead to the reactivation of TB (19). Other hypothesized mechanisms for the differing propensities to infection are differential binding of infliximab and etanercept to soluble and transmembrane TNF, and different binding kinetics (19). One effect of the latter finding could be granuloma breakdown while on infliximab because of complete neutralization of TNF and inflammatory cell recruitment, whereas etanercept would allow granuloma integrity because of less complete binding to TNF (19). Support for this hypothesis comes from the observation that etanercept has poor therapeutic effect in granulomatous inflammatory diseases such as Crohn's disease, Wegener's granulomatosis and sarcoidosis (20).

A number of other infections have been reported in patients receiving TNF- $\alpha$ antagonists. In their review of the AERS, Wallis et al (18) calculated an overall granulomatous infections adverse event rate of approximately 239 per 100,000 infliximab-treated patients, compared with approximately 74 per 100,000 etanercept-treated patients $(p<0.001)$. The most frequent pathogens observed, apart from M tuberculosis, were Histoplasma capsulatum, Candida species, Listeria species, nontuberculous Mycobacteria and Aspergillus species, the first five being more frequent in patients on infliximab. Less frequently reported and with equal frequency in infliximaband etanercept-treated patients were Cryptococcus, Nocardia, Salmonella, Toxoplasma, Brucella, Bartonella and Leishmania.

Histoplasmosis is the second most commonly reported infection in patients on TNF- $\alpha$ antagonists, which is not surprising given that it is the most common endemic mycosis in the US (16). One case series of 10 patients with $H$ capsulatum (21) reported that in the nine patients treated with infliximab, manifestations of infection occurred within 60 days in seven patients and within one week in another patient. All patients had received at least one other immunosuppressive medication and nine required aggressive treatment in an intensive care unit (21). These observations suggest that these infections may appear early in the course of treatment and may be associated with severe infection.

Serious bacterial infections have also been reported in patients on TNF- $\alpha$ antagonists $(22,23)$. In a case-control study of patients with RA who underwent an orthopedic surgical procedure (24), the prescription of a TNF- $\alpha$ antagonist was an independent risk factor for the development of a serious postoperative surgical site infection. Overall, the most common of the bacterial pathogens is Listeria monocytogenes (18). Estimated case rates for listeriosis within the first year of starting infliximab have been found to be approximately 6.1 per 100,000 patients compared with the overall US rate of 1.3 per 100,000 patients older than 60 years of age (25). Of the 15 patients in the AERS database identified as having an L monocytogenes infection, more patients had RA than Crohn's disease, 14 had received infliximab, 13 were on concomitant immunosuppressive therapies, and six died (25).

Other reports in the literature describe TNF- $\alpha$ antagonistassociated infections due to Pneumocystis jiroveci, Prototheca species, Coccidioides immitis, Sporothrix species, West Nile virus and hepatitis $B(7,8,26-28)$, with the potential for coinfections (29).

While the literature suggests an increased rate of infection in patients receiving TNF- $\alpha$ antagonists, the above studies fail to answer whether this is due to the drugs themselves or to other factors. To further explore this question, larger randomized, placebo-controlled trials, case-control studies and meta-analyses have been performed. Westhovens et al (30) conducted an international trial comparing two doses of infliximab with placebo in 1085 RA patients (followed for 54 weeks) already on methotrexate. Patients receiving placebo or $3 \mathrm{mg} / \mathrm{kg}$ infliximab had similar infection rates, whereas those receiving $10 \mathrm{mg} / \mathrm{kg}$ of infliximab as induction and maintenance had a threefold higher risk $(\mathrm{p}=0.013)$ of developing a serious infection (30). Westhovens et al concluded that the approved $3 \mathrm{mg} / \mathrm{kg}$ infliximab induction regimen (with later dose escalation allowed to control symptoms) was not associated with an increased rate of serious infections. In Germany, a cohort of patients with RA has been followed prospectively since May 2001 (31). At the time of follow-up in September 2004, 1529 patients had been entered into the database (601 control patients who were on conventional therapy, 512 etanercepttreated patients, 346 infliximab-treated patients and 70 anakinra-treated patients). Those patients on biological agents had more active RA than did the controls. In comparison 
with the control group, the incidence of serious infections was 2.7 to 2.8 times higher in patients receiving biological agents. The investigators acknowledged that more severe underlying disease may have been a contributing factor to the increased infection rate, but thought that the TNF- $\alpha$ antagonists were also playing a role (adjusted relative risks for etanercept and infliximab were 2.2 (95\% CI 0.9 to 5.4$)$ and 2.1 (95\% CI 0.8 to 5.5), respectively. Finally, a systematic review and metaanalysis incorporating 5014 patients randomly assigned to receive either anti-TNF therapy or a control treatment in nine trials was recently published (3). The risk of serious infections in patients with RA treated with anti-TNF antibodies (infliximab or adalimumab) was higher compared with placebo patients (OR 2.0; 95\% CI 1.3 to 3.1). This increased risk of infection was found for both high- and low-dose treatment groups as compared with placebo. For serious infections, the number needed to harm was 59 (95\% C1 39 to 125$)$ within a treatment period of three to 12 months.

Taken together, these studies suggest that TNF- $\alpha$ antagonists independently increase the risk of infection in patients who receive them. Of note, while opportunistic infections are the most exotic, the majority of infections are actually those more commonly seen, such as severe nontuberculous pulmonary infections, and skin and soft tissue infections, including herpes zoster $(3,30,31)$.

The use of biological treatments for inflammatory diseases is only expected to increase. Future directions are likely to include combined therapy with other cytokine antagonists, genetic evaluation of responsiveness to TNF antagonists, and optimizing dosing strategies (6). We can expect to see infections of varying severity as a side effect of these therapies.

In 2005, a group of rheumatologists from Europe, the US and Canada (convened to consider the use of biological agents) provided recommendations for reducing the risk of infection in an updated 2005 consensus statement on biological agents for the treatment of rheumatic diseases (32). Because most cases of TB in this population of patients with rheumatic diseases are likely due to reactivation, much importance is placed on screening patients for the possibility of latent TB. This includes a careful check of medical history, physical examination, tuberculin skin test (TST), and when indicated, a chest radiograph. The group recommended that treatment for latent TB be started before beginning TNF- $\alpha$ antagonists (32). A detailed history eliciting risks for TB is especially important, given the potential for a negative TST, particularly in patients with inflammatory bowel disease who have a high prevalence of anergy (33). Gardam et al (12) prefer completing the full course of treatment for latent TB before starting anti-TNF therapy. However, if the clinical condition warrants earlier treatment, it could be started one to two months after the start of isoniazid (12). In terms of other infectious complications, the authors recommend that TNF blocking agents should not be started or should be discontinued when serious infections occur, including septic arthritis, prosthetic infections, acute abscess, osteomyelitis, sepsis, systemic fungal infections, TB and listeriosis. Treatment should only be resumed if the infections have been treated adequately. Patients should be instructed to contact their physician if signs of serious infection occur. Patient education regarding food preparation and safety is important given the risk of Listeria and Salmonella infections (34). The consensus statement also recommends that pneumococcal and influenza vaccines be administered before starting the TNF- $\alpha$ inhibitors (32). Given the higher risk of postoperative infections, holding anti-TNF therapy before major surgery may be warranted (24). Finally, careful attention on the part of the treating physician to the development of infection in the patient is crucial.

It is unknown whether screening for coccidioidomycosis and histoplasmosis in patients living in endemic areas would be useful (34). One of the issues related to these two fungal infections is that most of these cases in immunocompromised hosts represent acute infection, making screening of little benefit (34). Similarly, whether antifungal prophylaxis would be of benefit is still to be determined. Also uncertain is whether asymptomatic patients with positive Histoplasma serology should be empirically treated with an antifungal agent before starting anti-TNF therapy, and if so, for how long.

There are little data on adherence to recommendations for preventing TB in patients on TNF- $\alpha$ inhibitor treatment and on whether those measures are effective. A survey in April 2005 , answered by 1023 rheumatologists in the US, found that while $92 \%$ of respondents felt that a TST was indicated when using a TNF inhibitor, only $59.1 \%$ actually performed this test on their patients (35). On the other hand, simple steps to prevent the reactivation of latent TB appear to work. The risk of active TB among Spanish patients taking TNF antagonists decreased by $78 \%$ (RR $0.22 ; 95 \%$ CI 0.03 to 0.88 ) after the official recommendations on detecting and treating latent TB in this patient population were issued (36). Reductions in TB were also found after the introduction of education/screening programs with adalimumab and infliximab (17).

Biological therapies have made important advances in the treatment of a number of inflammatory diseases. They have also given insight into the role of various cytokines in host defense. We are in the early stages of understanding the magnitude and spectrum of infectious complications associated with these therapies. Much work is needed on developing evidence-based guidelines related to prevention and management of infections in these patients, including the withholding of biological therapies.

\section{REFERENCES}

1. Phend C. Biologics continue to look promising for Crohn's disease. $<$ http://www.medicalpost.com/medicine/clinical/article.jsp?content= 20060612_170650_5540> (Version current at July 24, 2006).

2. Olsen NJ, Stein CM. New drugs for rheumatoid arthritis. N Engl J Med 2004;350:2167-79.

3. Bongartz T, Sutton AJ, Sweeting MJ, Buchan I, Matteson EL, Montori V. Anti-TNF antibody therapy in rheumatoid arthritis and the risk of serious infections and malignancies: Systematic review and meta-analysis of rare harmful effects in randomized controlled trials. JAMA 2006;295:2275-85. (Erratum in 2006;295:2482).

4. Elliott MJ, Maini RN, Feldmann M, et al. Repeated therapy with monoclonal antibody to tumour necrosis factor alpha (CA2) in patients with rheumatoid arthritis. Lancet 1994;344:1125-7.

5. Siddiqui MA, Scott LJ. Infliximab: A review of its use in Crohn's disease and rheumatoid arthritis. Drugs 2005;65:2179-208.

6. Weaver AL. Differentiating the new rheumatoid arthritis biologic therapies. J Clin Rheumatol 2003;9:99-114.

7. Rychly DJ, DiPiro JT. Infections associated with tumor necrosis factoralpha antagonists. Pharmacotherapy 2005;25:1181-92.

8. Khoury JA, Dubberke ER, Devine SM. Fatal case of protothecosis in a hematopoietic stem cell transplant recipient after infliximab treatment for graft-versus-host disease. Blood 2004;104:3414-5.

9. Krueger G, Callis K. Potential of tumor necrosis factor inhibitors in psoriasis and psoriatic arthritis. Arch Dermatol 2004;140:218-25.

10. Weaver AL. Efficacy and safety of the anti-TNF biologic agents. Mod Rheumatol 2004;14:101-12. 
11. Nestorov I. Clinical pharmacokinetics of tumor necrosis factor antagonists. J Rheumatol Suppl 2005;74:13-8.

12. Gardam MA, Keystone EC, Menzies R, et al. Anti-tumour necrosis factor agents and tuberculosis risk: Mechanisms of action and clinical management. Lancet 2003;3:148-55.

13. Ehlers $\mathrm{S}$. Why does tumor necrosis factor targeted therapy reactivate tuberculosis? J Rheumatol Suppl 2005;74:35-9.

14. Mufti AH, Toye BW, Mckendry RR, Angel JB. Mycobacterium abscessus infection after use of tumor necrosis factor alpha inhibitor therapy: Case report and review of infectious complications associated with tumor necrosis factor alpha inhibitor use. Diagn Microbiol Infect Dis 2005;53:233-8.

15. Keane J, Gershon S, Wise RP, et al. Tuberculosis associated with infliximab, a tumor necrosis factor alpha-neutralizing agent. N Engl J Med 2001;345:1098-104.

16. Hamilton CD. Immunosuppression related to collagenvascular disease or its treatment. Proc Am Thorac Soc 2005;2:456-60.

17. Keystone EC. Safety of biologic therapies - an update. J Rheumatol Suppl 2005;74:8-12.

18. Wallis RS, Broder MS, Wong JY, Hanson ME, Beenhouwer DO. Granulomatous infectious diseases associated with tumor necrosis factor antagonists. Clin Infect Dis 2004:38:1261-5.

19. Ehlers S. Tumor necrosis factor and its blockade in granulomatous infections: Differential modes of action of infliximab and etanercept? Clin Infect Dis 2005;41(Suppl 3):S199-203.

20. Wallis RS, Broder M, Wong J, Lee A, Hoq L. Reactivation of latent granulomatous infections by infliximab. Clin Infect Dis 2005;41(Suppl 3):S194-8.

21. Lee JH, Slifman NR, Gershon SK, et al. Life-threatening histoplasmosis complicating immunotherapy with tumor necrosis factor alpha antagonists infliximab and etanercept. Arthritis Rheum 2002;46:2565-70.

22. Kroesen S, Widmer AF, Tyndall A, Hasler P. Serious bacterial infections in patients with rheumatoid arthritis under anti-TNFalpha therapy. Rheumatology (Oxford) 2003;42:617-21.

23. Maillard H, Ornetti P, Grimault L, et al. Severe pyogenic infections in patients taking infliximab: A regional cohort study. Joint Bone Spine 2005;72:330-4.

24. Giles JT, Bartlett SJ, Gelber AC, et al. Tumor necrosis factor inhibitor therapy and risk of serious postoperative orthopedic infection in rheumatoid arthritis. Arthritis Rheum 2006;55:333-7.

25. Slifman NR, Gershon SK, Lee JH, Edwards ET, Braun MM. Listeria monocytogenes infection as a complication of treatment with tumor necrosis factor alpha-neutralizing agents. Arthritis Rheum 2003;48:319-24.

26. Chan-Tack KM, Forrest G. West nile virus meningoencephalitis and acute flaccid paralysis after infliximab treatment. J Rheumatol 2006;33:191-2

27. Millonig G, Kern M, Ludwiczek O, Nachbaur K, Vogel W. Subfulminant hepatitis B after infliximab in Crohn's disease: Need for HBV-screening? World J Gastroenterol 2006;12:974-6.

28. Esteve M, Saro C, Gonzalez-Huix F, Suarez F, Forne M, Viver JM. Chronic hepatitis B reactivation following infliximab therapy in Crohn's disease patients: Need for primary prophylaxis. Gut 2004;53:1363-5.

29. van der Klooster JM, Bosman RJ, Oudemans-van Straaten HM, van der Spoel JI, Wester JP, Zandstra DF. Disseminated tuberculosis, pulmonary aspergillosis and cutaneous herpes simplex infection in a patient with infliximab and methotrexate. Intensive Care Med 2003;29:2327-9.

30. Westhovens R, Yocum D, Han J, et al; START Study Group. The safety of infliximab, combined with background treatments, among patients with rheumatoid arthritis and various comorbidities: A large, randomized, placebo-controlled trial. Arthritis Rheum 2006;54:1075-86.

31. Listing J, Strangfeld A, Kary S, et al. Infections in patients with rheumatoid arthritis treated with biologic agents. Arthritis Rheum 2005;52:3403-12.

32. Furst DE, Breedveld FC, Kalden JR, et al. Updated consensus statement on biological agents, specifically tumour necrosis factor alpha (TNF alpha) blocking agents and interleukin-1 receptor antagonist (IL-1ra), for the treatment of rheumatic diseases, 2005. Ann Rheum Dis 2005;64(Suppl 4):iv2-14.

33. Mow WS, Abreu-Martin MT, Papadakis KA, Pitchon HE, Targan SR, Vasiliauskis EA. High incidence of anergy in inflammatory bowel disease patients limits the usefulness of PPD screening before infliximab therapy. Clin Gastroenterol Hepatol 2004;2:309-13.

34. Crum NF, Lederman ER, Wallace MR. Infections associated with tumor necrosis factor-alpha antagonists. Medicine (Baltimore) 2005;84:291-302.

35. Cush JJ. Biological drug use: US perspectives on indications and monitoring. Ann Rheum Dis 2005;64(Suppl 4):iv18-23.

36. Carmona L, Gomez-Reino JJ, Rodriguez-Valverde V, et al; BIOBADASER Group. Effectiveness of recommendations to prevent reactivation of latent tuberculosis infection in patients treated with tumor necrosis factor antagonists. Arthritis Rheum 2005;52:1766-72. 


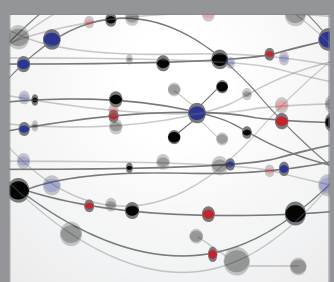

The Scientific World Journal
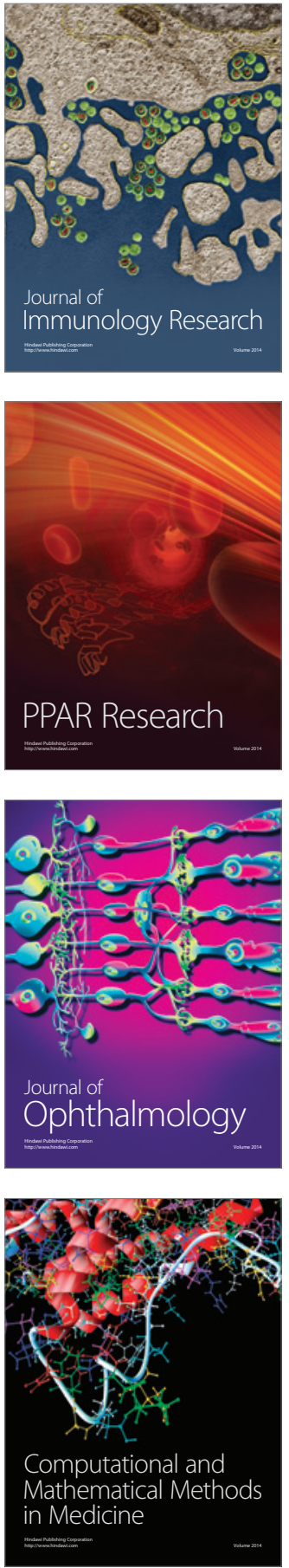

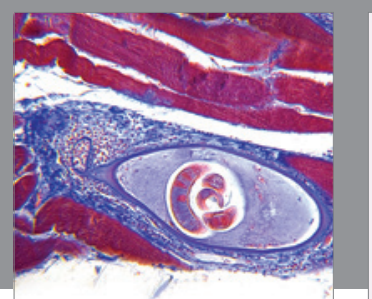

Gastroenterology Research and Practice

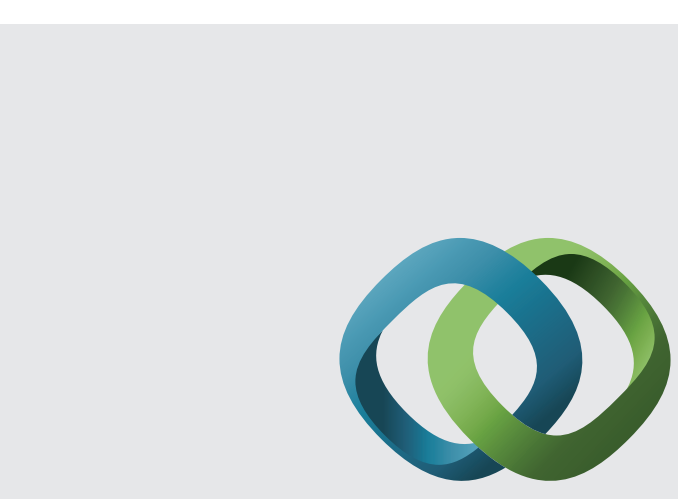

\section{Hindawi}

Submit your manuscripts at

http://www.hindawi.com
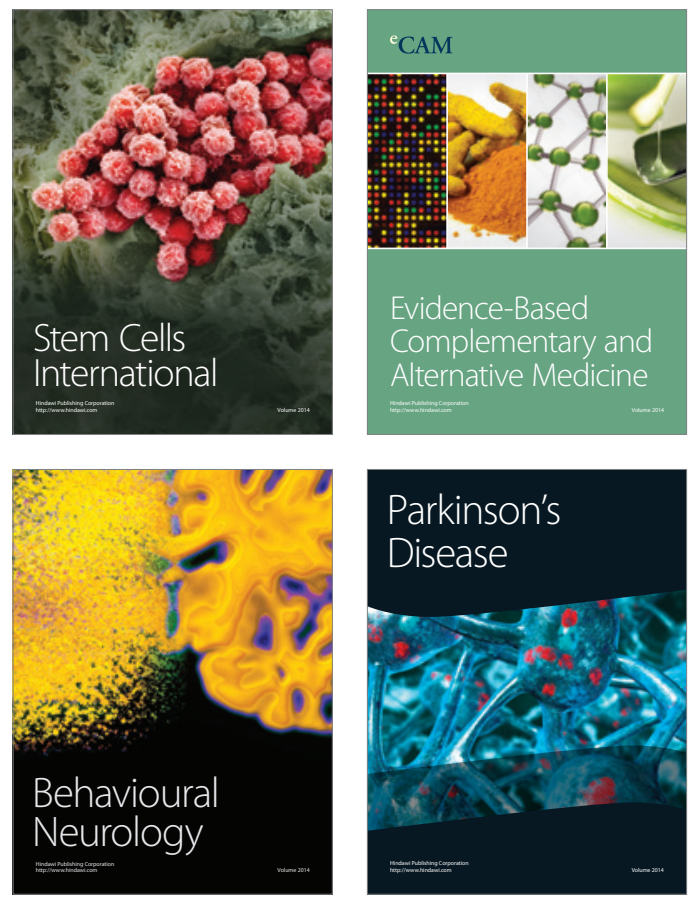
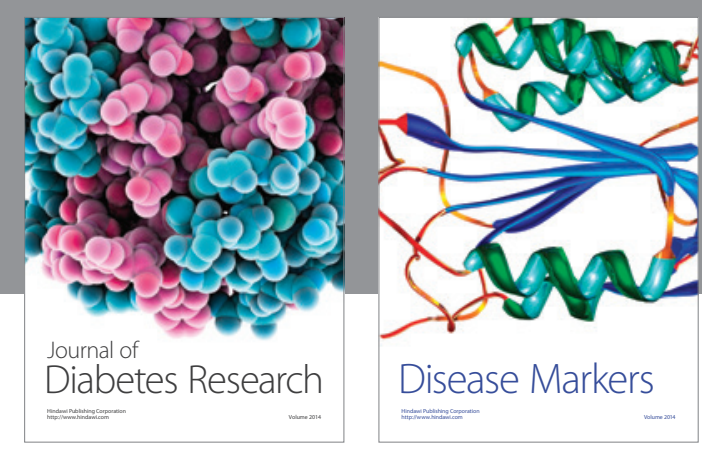

Disease Markers
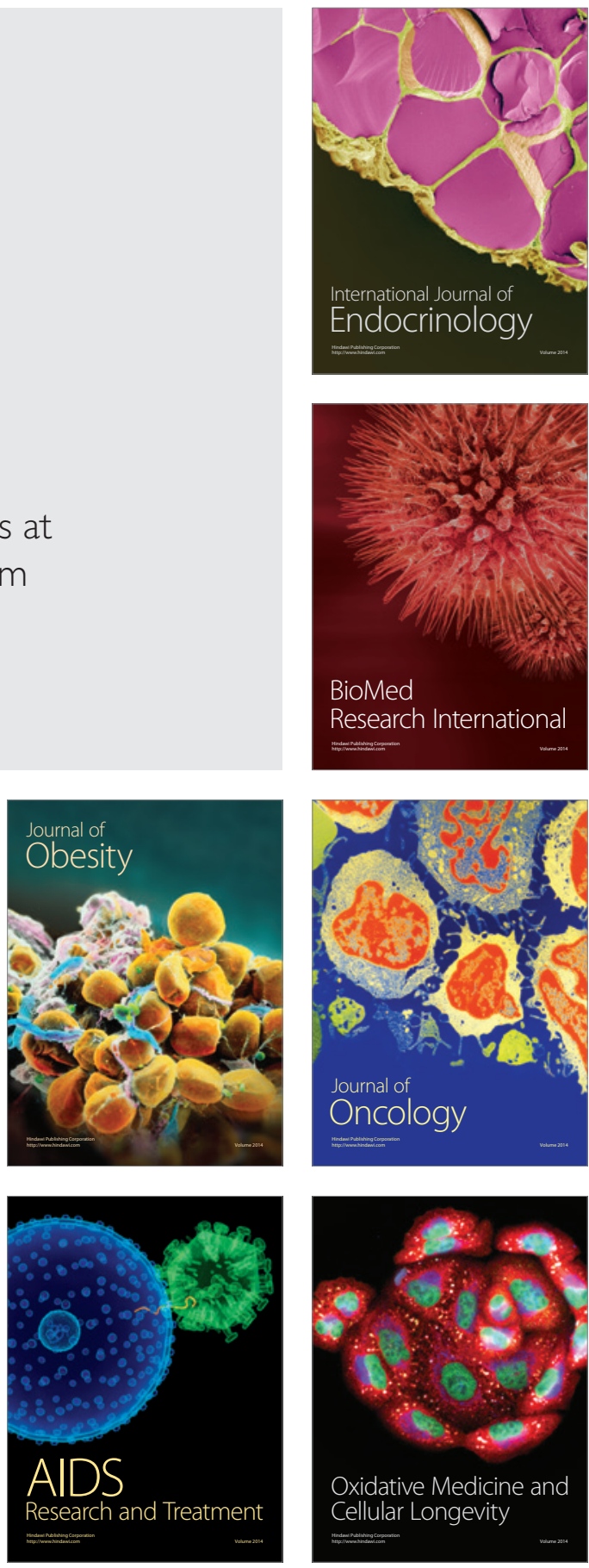